\title{
GuideME: AN EFFECTIVE RFID-BASED TRAFFIC MONITORING SYSTEM
}

\author{
Fadi Aloul, Assim Sagahyroon, Ali Nahle, Makram Abou Dehn, Raneem Al Anani \\ American University of Sharjah \\ Sharjah, United Arab Emirates (UAE) \\ \{faloul, asagahyroon, b00019676, b00019760,g00021346\}@aus.edu
}

\begin{abstract}
Traffic jam is a serious problem that has been around for a very long time. In Project GuideME, we propose a system that monitors the highways and calculates the average speed of the passing cars before storing the data in a database. The user can then access real-time updates with exact numerical values of the highways' average speed via a web portal or SMS. Therefore, users can plan the fastest routes for their trips, and reduce congestion by distributing traffic better over the highways. The solution uses two Radio Frequency Identification (RFID) readers on each monitored highway, and installs passive RFID tags on the passing cars. The two RFID readers are integrated with a General Packet Radio Service (GPRS) device that sends data to a web server. The server runs a calculating algorithm and a database to store the data. A prototype was built and tested. It operated as intended, was relatively cheap to implement and delivered accurate results. With the promising preliminary results, we believe that GuideME would function very well in a reallife and large-scale implementation, and can benefit a large number of people.
\end{abstract}

\section{KEY WORDS}

Real Time Traffic Monitoring, RFID, GPRS, Web Server, Google Maps.

\section{Introduction}

In 2000, BBC reported that pollution due to road traffic is to be blamed for the death of tens of thousands every year across Europe [1]. Another research published in The Lancet Medical Journal estimated that $6 \%$ of deaths in several countries in Europe were due to air pollution; where half of these deaths (around 20,000) were linked to toxic fumes emitted from vehicles stuck in traffic [2]. Furthermore, the researchers have also estimated that traffic fumes were responsible for more than 25,000 new cases of chronic bronchitis and more than 500,000 asthma attacks.

In 2007, MSNBC reported a U.S. study stating that traffic pollution is affecting children who live near busy roads by preventing their lungs from developing properly [3]. As a result, this makes them more vulnerable to suffer respiratory and heart problems later in life. According to the American Lung Association, air pollution due to traffic can seriously contribute to premature death, lung cancer, chronic bronchitis and heart attacks. Furthermore, it can also lead to asthma attacks, coughing, wheezing, shortness of breath, coughing, wheezing and other respiratory and cardiovascular diseases.

In Ealing, UK, the major source of air pollution was identified to be the fumes produced from vehicles in road traffic [4]. Despite the attempts of making vehicle emissions cleaner, yet the continuous increase in road traffic could seriously suggest that the pollution may continue to rise in the future.

The traffic jam problem is unfortunately an on-going old problem that is not showing any signs of improvement. This problem is resulting in (1) fatal accidents due to the created stress, (2) health and environment problems due to increasing toxic gas emissions from vehicles, (3) noise pollution, (4) late arrival at work or schools due to the time people spend while they are stuck in traffic, (5) late arrival of emergency vehicles due to traffic jam, and much more. This paper aims at reducing the traffic jam problem and creating a more sustainable and healthy environment.

Several solutions have been previously proposed to solve the traffic jam problem. Those solutions vary in their core technology; as some of them use CCTV cameras and image processing, GPS and satellites, or GSM and cellular towers. However, many of those solutions are either very expensive or not very effective.

In this work, we propose to use the Radio Frequency Identification (RFID) technology to solve the traffic jam problem. The main reason behind preferring RFID technology over others is in the relatively low cost of RFID gear and the variety of available options. RFID has also been successful in solving a wide span of problems in various domains $[5,6,7,8]$.

The remaining paper is divided as follows. Section 2 presents a background of RFID and general equipment needed for the proposed solution. Section 3 presents a description of the proposed solution. Section 4 presents the results of testing the proposed solution. Section 5 compares the proposed solution to existing work. The paper is concluded in Section 6.

\section{Background}

The following section presents a description of the equipment needed for the proposed solution.

\subsection{RFID}


Radio frequency identification (RFID) is a system that transmits the identity of an object using radio waves. This identification is transmitted in the form of a serial number that distinguishes each object from the other. The RFID system is composed of a RFID reader and a Tag. The tag is composed of a microchip connected to an antenna; microchip can store a maximum of 2 kilobyte of data, which can include information about the product, the manufacturing date, the destination, among other information. In order to retrieve the data stored in the tag we need a reader, which is a device that emits radio waves, these radio waves are received by the tag which activates the microchip and then the data get transmitted.

The tags can be distinguished by their mode of operation as follows:

Read Only Tags - This type of tags has information stored on them during the manufacturing process, and this information cannot be changed.

Read/Write Tags - This type of tags has separate writable memory areas. This is a very important feature because the information contained in the microchip can be written and after that changed, especially if the information changes overtime [5].

WORM Tags - WORM tags stands for Write Once Read Many. The information contained in this tag can only be changed once, but can be read as many times.

The RFID tags contains two parts, one part is the integrated circuit for storing and processing the data, modulating and demodulating radio frequency signal, and the other part is the antenna for receiving and transmitting the signal. These tags can operate on many levels of frequency ranging from $125 \mathrm{KHz}$ until $915 \mathrm{MHz}$. There are three main types of RFID tags, which are:

Passive Tags - The tags have no internal power supply; therefore the microchip is in sleep mode, until the tag is in the range of the reader, where the reader sends electromagnetic waves, these waves "wake up" the microchip where it converts the waves into digital data using modulators and send it to the reader. This type of tags is usually cheaper than other tags with the price ranging from U.S. 20 cents to several dollars, depending on the amount of memory in the tag and the packaging details [9]. The read range of the tag is typically between 0 to 5 meters, however, recently a new passive ultrahighfrequency (UHF) RFID tag, known as the S/Cap tag, has been developed with claims that it can be read from a distance of 25 meters. The new tag uses small solar panels to perform like a battery-assisted tag and costs between $\$ 12$ to $\$ 15$ [10]. Passive tags can be used with fastmoving objects, for example the automatic train identification system (ATIS) in China uses passive tags to detect railway cars travelling at a speed of $150 \mathrm{kph}$ [11]. In United Arab Emirates, toll gates, known as Salik gates, use RFID readers to detect passive tags installed on fastmoving car windshields from a distance of 5 meters [12]. Note that the Salik system has been shown to be successful in detecting cars travelling at a speed of 200+ kph. Figure 1 shows an example of how the Salik system works and Figure 2 shows a sample Salik toll gate.

Active Tags - The tags have their own internal power supply, which is used to power the integrated circuits and to transmit the data to the reader. Communications from active tags to readers is typically much more reliable than the communications from passive tags to readers. In addition to the power supply the active tag can have onboard electronics such as sensors, I/O ports among others that are powered by the on-board power supply. With this feature the active tags can be used in much more applications than the passive tags. The read range of the tag can reach 100 meters.

Semi-Passive Tags - The tags lay between the passive and the active tags, because they have a battery to power the integrated circuit however they use the power from the electromagnetic waves that the reader sends in order to retrieve the data present on the tag.

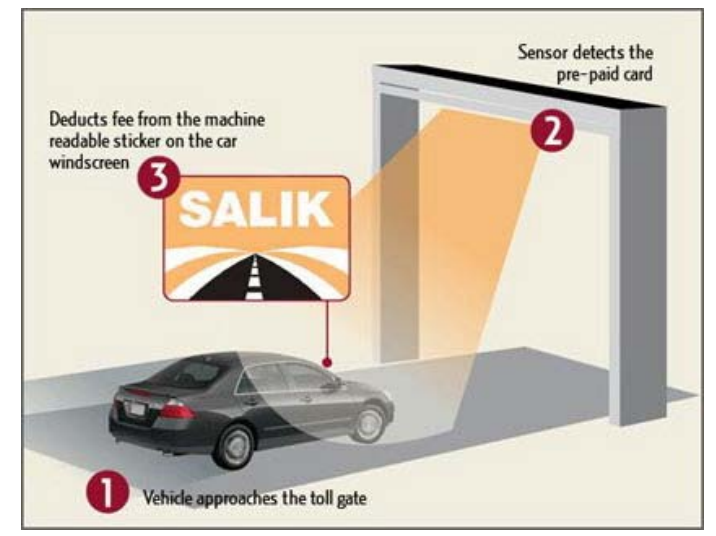

Figure 1. Salik toll gate system procedure [12].

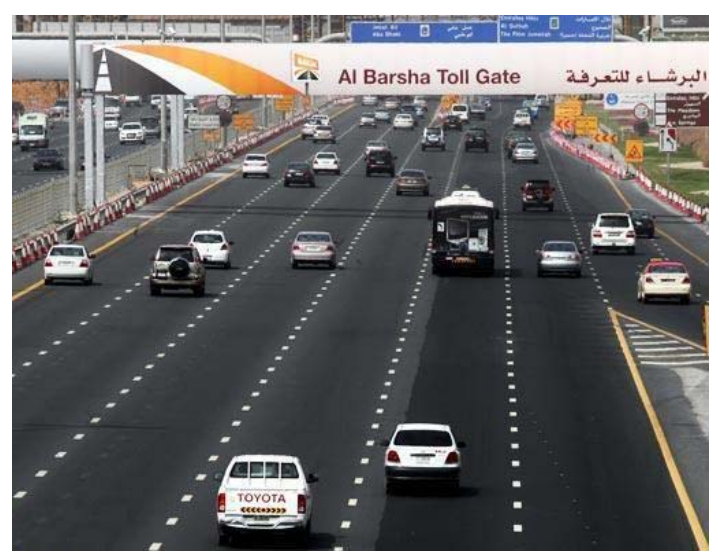

Figure 2. Sample Salik toll gate [12]. 


\subsection{GPRS}

General Packet Radio Service (GPRS) is a packet based wireless system that can send data from 56 up to 114kbps. Because it is a packet switched based communication, it costs the user less than circuit switched communication. Channels are shared among user and no dedicated channels for each user. This help to make the connection more efficient and sending data will be faster. Though the theoretical maximum is $171.2 \mathrm{Kbps}$, typical throughput is up to 20 or $30 \mathrm{Kbps}$.

There are many advantages for using GPRS:

- Real time data transfer.

- The user is charged on the amount of data sent not on the time used to transfer data as it is the case for GSM services.

- Packets from one user will be sent via different time slots.

- User can share time slots.

- User will be always connected even if he is not sending any data.

- It is within the GSM standard so no need to use new frequencies.

\subsection{Google Maps API}

The Google Maps API allows web sites to embed the Google maps into the web page by including a specific JavaScript code. The API grants the power to manipulate the maps, add content to them, and create a custom built maps application. The Maps API is available for free use to consumers for personal use. In this work, Google maps APIs will be used to represent points of congestion on the maps.

The Google Map code is composed almost entirely of JavaScript and XML. JavaScript is a popular scripting language that is used in web-page development and is very similar to syntax to JAVA. Through JavaScript, we can write functions to get access to objects that are embedded in the application. This allows the application to be more responsive to the user's input. XML stands for Extensible Markup Language, and it is used to create general markup languages.

To use the Google Maps API, we have to request an API Key that is bound to our website. Since we want to plot the points of our congestion points on the map, we will create our own map interface by first adding the Google JavaScript code to our page's code, and then writing or using JavaScript functions to plot the points onto the map. Furthermore, Google Maps can be viewed on several modern mobile phones; such as the iPhone, BlackBerry, and the Nokia S60 3rd edition series. Moreover, Google Maps API adds support for Mapplets; which is Maps + Gadgets. Basically, you can host your application on a website and use the Mapplets to embed this hosted application within Google Maps.

\section{Design Architecture}

The goal of the proposed design, named as GuideME, is to represent a simple, inexpensive, and effective solution that provides real-time numerical values of the highways' average speed. The design consists of two components: (1) a server stationed at the Department of Road and Transportation, and (2) a client placed at various highway locations that are to be monitored. The server typically consists of a web server and a GSM modem that can be used to send SMS alerts. The client consists of two RFID readers and a GPRS modem. The overall system architecture is shown in Figure 3.

For each monitored location, the two RFID readers are placed on the side of the highways with a fixed short distance in between, typically 100 meters. We assume that most cars will have a passive RFID tag installed on them. The RFID readers will detect a passing car by reading the passive tag installed on it. The two passing times, with the corresponding tag number are recorded and transmitted via the GPRS modem to the central web server in the Department of Road and Transportation. On the web server, a running algorithm calculates the speed of the passing car (i.e. divides the difference of the two recorded times by 100 meters) and stores the computed speed in the database. As more cars pass by, the average speed of the highway becomes more accurate.

As shown in Figure 1, a user can then identify, in real-time, the average speed of the passing cars on the monitored highways using two convenient options: (1) SMS or (2) web portal. Using either way, the users can get to know which highways are congested and which are not, and therefore can better plan their route and avoid the congested roads. Users with internet access can look up the speed using the GuideME website. An example of a screenshot is shown in Figure 4. The SMS option is available for users who don't have access to an internet connection. An example of a mobile screenshot is shown in Figure 5. The web portal allow users to create personal profiles and set customized SMS/Email alerts for specific roads to stay up-to-date with the traffic conditions. The portal will automatically notify the user when specific conditions occur on the selected monitored roads.

In terms of privacy, the only data read from the passing cars is the RFID tag number. No license plates, car details, or driver information will be associated with the tag numbers and hence the driver privacy should be maintained. Nevertheless, some users might still refuse to install a tag for privacy issues which is acceptable, since the system needs only a sample of registered cars to get the average speed. A simple solution is to install the tags on public transportation vehicles, such as taxis, which should be enough to identify the average speeds on each monitored point.

In addition to staying up-to-date with the road conditions, GuideME keeps an archive of all the collected data, and can provide clear illustrative graphs to help researchers, engineers, or interested citizens view the 
traffic patterns. The data can be used by traffic researchers to study the traffic conditions and improve the flow.

The proposed system attempts to achieve its objectives using minimal components to reduce cost and ease maintenance. Table 1 lists the equipment used in the proposed design and their corresponding cost. The total cost per location is approximately $\$ 1500$ assuming two RFID readers only. Note that for highways with many lanes, more than two RFID readers might be needed to detect all passing vehicles which may increase the cost per location. However, if the budget is limited, the system can still work using the two readers as they can be installed on the fast lane of the highway. Each registered car will need a passive RFID tag which will cost approximately $\$ 10$.

In terms of running cost for sending the data via GPRS, GuideME proves to be cost-effective as shown in Table 2. The charges shown in Table 2 are for the UAE's telecommunication company. However, other telecommunication companies charge a flat rate for unlimited data usage which can be yet cheaper than what is shown in Table 2.

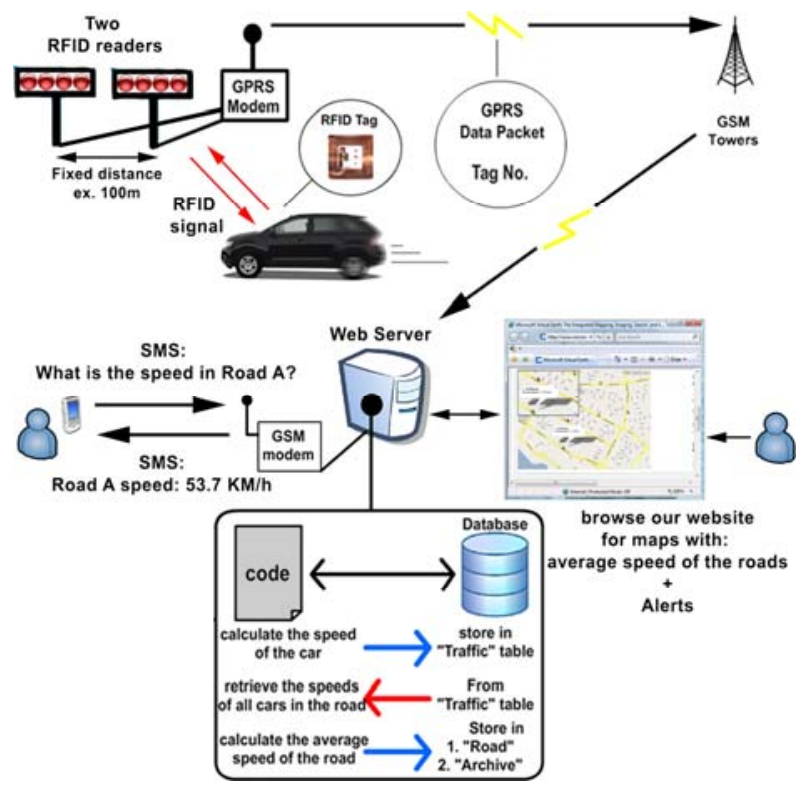

Figure 3. Overall system architecture.

\section{Experimental Results}

A prototype was built and tested with different scenarios. Overall, the prototype operated well and as intended. Three cars, equipped with the passive tags on their windshields, were used to test the proposed system while moving at speeds of 10,25 , and $40 \mathrm{kph}$, respectively. The used Texas Instruments RFID readers were able to detect the passing passive tags at a distance of 3 meters, and the speed of each tag was successfully calculated. The RFID readers sent the two passing times with the corresponding tag number in a packet of size 64 bits. The average speed was then successfully computed on the web server and accurately mapped onto the Google Maps. The Siemens GSM modem that was connected to the web server was successful in answering the requests sent to it via SMS and replying back to the user with the average speed of the requested road.

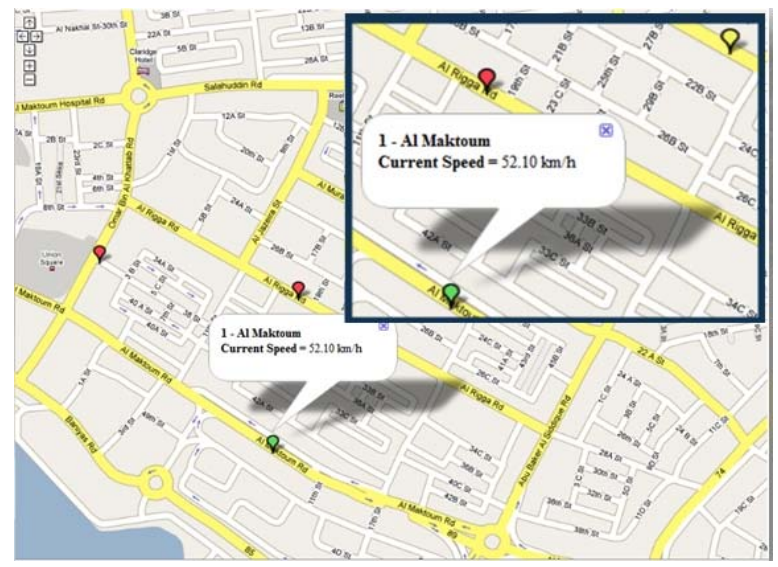

Figure 4. Web portal with speed maps.

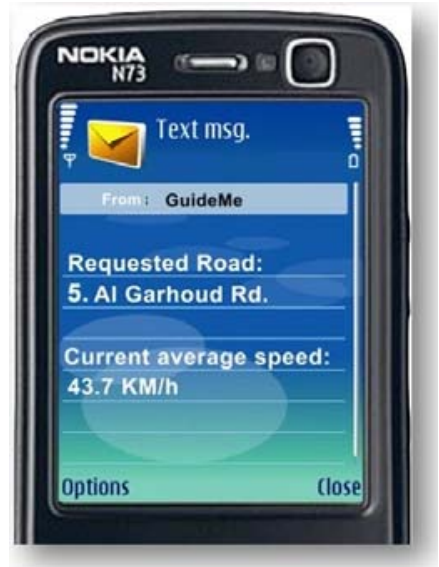

Figure 5. Retrieve speed information via SMS.

\section{Existing Solutions}

Several solutions have been proposed to monitor and reduce the traffic jam problem, some of which are RFID based and others rely on other advanced technologies. In this section, we summarize some of the existing work and compare it against the proposed model.

\section{1 mGate}

In the year 2006, the New York State Department of Transportation and a number of transportation researchers at Rensselaer Polytechnic Institute worked on a project to develop a solar-powered mobile RFID reader that reads EZPass tags installed on the passing cars [13]. The tags 
are originally used to wirelessly pay for bridge and highway tolls. The new approach provided a method of monitoring traffic while using solar energy to power the readers if they are installed on roads that are under construction or under other special circumstances. The RFID reader device, codenamed $m$ Gate, was connected to a laptop computer via a USB port that used solar-powered batteries. Afterwards, the laptop would send the encrypted tag ID, timestamp, and reader's location over a wireless internet connection to a central server.

The system's objective was to calculate the time taken by traffic to move from one RFID reader to another. Based on the duration of the trip, the system could alert the travelling drivers about slow traffic via electronic signage on the road or the Internet.

In comparison to our proposed solution, this system places only one RFID reader on each road; while our solution places two RFID readers on each road that are separated by a short fixed distance (100-200 meters only). In mGate, the distance between the RFID devices is several miles, and the speed of the passing vehicles is most likely to vary greatly over that distance due to different road conditions; which affects the trip's time that the system depends on to calculate the speed. Therefore, the calculated speed would not be accurate. Furthermore, this system requires a laptop to be connected to every RFID reader; which introduces a high cost to every monitored location. Also, the system provides its data to the citizens through signage and the internet only, while GuideME provides data to the user in the form of graphical maps and through SMS.

\subsection{Tollgates and RFID}

Another RFID-based solution was developed back in the year 2004 by the Orlando-Orange County Expressway Authority (OOCEA) in Florida-USA [14]. The system used toll-collection transponders to calculate the travel time of individual cars as they pass from one reader to another so that an average time could be calculated and then provided to the public. It implemented RFID readers on the roadside to read signals sent from the transponders installed on cars; where those transponders were originally intended for the use of automatic toll payment methods in central Florida. The data was then sent to Florida Department of Transportation (FDOT) and OOCEA; who would later provide it to the public through electronic signage installed on the roads. Another method for the user to gain access to the data was by calling the National Travel Information number or by accessing a web site.

In contrast to our design, this system is very similar to the previously discussed mGate system in New York. Again, it depended on one single RFID reader on each road and on the time between each consecutive tollgate to calculate the speed. Those tollgates are typically several miles apart, and the speed is most likely to vary greatly when moving from one tollgate to the other. Therefore, the calculated data is subject to a high probability of inaccuracy, especially if a car stops or slows in between the RFID readers.

\begin{tabular}{|c|c|c|c|}
\hline Equipment & Quantity & $\begin{array}{c}\text { Unit Price } \\
\text { (US \$) }\end{array}$ & $\begin{array}{c}\text { Total Price } \\
\text { (US \$) }\end{array}$ \\
\hline $\begin{array}{c}\text { RFID Reader } \\
\text { (Texas Instruments) }\end{array}$ & 2 & $\$ 500$ & $\$ 1000$ \\
\hline $\begin{array}{c}\text { GPRS Modem } \\
\text { (ComTech) }\end{array}$ & 1 & $\$ 300$ & $\$ 300$ \\
\hline $\begin{array}{c}\text { Microcontroller } \\
\text { (HCS12) }\end{array}$ & 1 & $\$ 200$ & $\$ 200$ \\
\hline \multicolumn{2}{|c|}{ Total Cost Per Location } & $\$ 190$ & $\$ 190$ \\
\hline \begin{tabular}{c|c|c|} 
GSM Modem \\
at Server (Siemens)
\end{tabular} & 1 & $\$ 10$ & $\$ 10 /$ vehicle \\
\hline Passive RFID Tag & $\begin{array}{c}\text { Per } \\
\text { vehicle }\end{array}$ & $\$$ \\
\hline
\end{tabular}

Table 1. Required equipment and cost.

\begin{tabular}{|c|c|c|c|}
\hline & $\begin{array}{c}\text { Light } \\
\text { Traffic }\end{array}$ & $\begin{array}{c}\text { Medium } \\
\text { Traffic }\end{array}$ & $\begin{array}{c}\text { Heavy } \\
\text { Traffic }\end{array}$ \\
\hline $\begin{array}{c}\text { Expected } \\
\text { Cars per day }\end{array}$ & 1,000 & 5,000 & 10,000 \\
\hline $\begin{array}{c}\text { Packet Size } \\
\text { per car }\end{array}$ & 64 Bits & 64 Bits & 64 Bits \\
\hline $\begin{array}{c}\text { Transmitted } \\
\text { Data }\end{array}$ & $64 \mathrm{~K}$ Bits & $320 \mathrm{~K}$ Bits & $640 \mathrm{~K}$ Bits \\
\hline $\begin{array}{c}\text { Cost } \\
\mathbf{( \$ 0 . 0 0 1 / K B )}\end{array}$ & $\$ 0.064$ & $\$ 0.32$ & $\$ 0.64$ \\
\hline
\end{tabular}

Table 2. Sample running cost.

\subsection{CarTel}

Drifting away from RFID-based traffic monitoring solutions, from the Massachusetts Institute of Technology (MIT) developed a system codenamed CarTel [15]. The objective of CarTel was to recommend better routes for the drivers. The system created a mobile-sensing network by installing a very small computer device on moving vehicles. The device calculated the speed of its vehicle at certain intervals and sent it via wireless Internet to a database. The web portal provided geo-spatial data visualization maps that represented the data received from the sensors on the cars. 
Compared to GuideME, the system CarTel depends on Wi-Fi networks that the vehicles pass by on the way of the trip to send the data. This method wouldn't work in the numerous numbers of countries where there aren't many Wi-Fi spots. Our solution uses GPRS to send the data; which ensures that data can be virtually sent anytime and anywhere.

\subsection{Cellular Towers}

Another system was developed in India that used cellular towers to get an indication of the congestion levels [16]. The system detects traffic congestion based on the level of cell phone congestion. Basically, more users meant higher congestion. A number of micro-towers were installed in certain areas at traffic crossings. By sending cell phone signals to detect cell phone congestions, it can obtain realtime pattern of the movements at different location between the towers. The data was placed on a website where users could retrieve the congestion's information through SMS.

However, this solution does not give numeric speed values; requires high data transmission back and forth between the towers and the cell phones; and also requires erecting several expansive monitoring micro-towers in areas with no coverage or with many users.

\subsection{Image Processing}

Image processing has been used for a long time to monitor traffic [17]. In such a solution, the data is first provided in a video sequence. The video is then processed to locate the observed vehicles in each frame of the video. Several image processing techniques are applied; such as segmentation, filtering and edge detection. This allocates the vehicle contour within the frame observation zone. To calculate the speed of a vehicle, the conditional centre of the vehicle must be found in the contour area. By labeling and counting the contours, we can calculate the number of moving vehicles in the observed zone; therefore, we can estimate the level of road's congestion in that area.

Nevertheless, this solution depends solely on implementing a large number of expensive Closed Circuit Television (CCTV) cameras around the city; not to mention the expensive equipment required to process the data and the long time taken to process the frames.

\subsection{Satellite and GPS}

Another common solution to the world of traffic monitoring uses GPS and satellites. In such a system, the road traffic is monitored via a satellite link. Satellites offer wide coverage and can track the vehicles through a telecommunications module installed on the vehicle. This installed device obtains the vehicle's velocity and position data using a GPS receiver, and transmits the data to a hub station. The hub station sends the data to related parties. The hub regularly broadcast a message to all vehicles requesting them to send their data to build an up-to-date database of the speed and location of the vehicles. The identity of the vehicle's owner is kept anonymous be generating new random ID each time they join the system. However, implementing this system is expensive as it requires in-car GPS system in every vehicle to calculate the speed, and also requires an additional telecommunications module for data transfer. It is worth noting as well that this system is highly affected by weather conditions and does not function in tunnels.

\section{Conclusion}

Based on the experimental results we obtained, GuideME shows very promising potential. The system has a significant edge in terms of cost and effectiveness in comparison to the other non-RFID based traffic monitoring solutions. The image processing solution requires a large number of expensive CCTV cameras and powerful equipment to process the captured frames. The GPS and satellites solution requires expensive in-car GPS system and additional telecommunication module; not to mention that it does not work inside tunnels. Also, the other system that uses cellular towers does not give numeric speed values, takes a lot of valuable transmission bandwidth, and requires erecting several expensive monitoring micro-towers. Even when compared to the other RFID based solutions, GuideME proves to yield accurate results while cutting down the costs. To summarize, GuideME proves to be both effective and inexpensive traffic monitoring solution.

Furthermore, the system shows high potential for extendibility. For example, cars could synchronize their GPS navigation systems with the GuideME server to provide the road conditions on the fly. Also, GuideME could even replace the expensive speeding RADAR. We strongly believe that a real-life implementation of the system on a larger scale would function as intended and benefit a large number of people.

\section{References}

[1] "Traffic Pollution Kills Thousands," BBC News, 2000. Available at: http://news.bbc.co.uk/2/hi/health/ 905016.stm.

[2] "Cutting Greenhouse Gas Emissions has Major Direct Health Benefits," The Lancet Medical Journal, December 2009. Available at: http://climateprogress.org/2009/12/01/the-lancetmedical-journal-cutting-greenhouse-gas-emissions-hasmajor-direct-health-benefits/.

[3] "Road Pollution Can Damage Kid's Lungs, Hearts," MSNBC Health, 2007. Available at: http://www.msnbc. msn.com/id/16831975/.

[4] "Road Traffic," Available at: http://www.ealing.gov.uk/services/environment/pollution/ air_pollution/road_traffic. 
[5] Klaus Finkenzeller, "RFID Handbook: Fundamentals and Applications in Contactless Smart Cards, Radio Frequency Identification and Near-Field Communication,” Wiley, 2010.

[6] B. Nath, F. Reynolds, R. Want, "RFID Technology and Applications," IEEE Pervasive Computing, 5(1), 2224, 2006.

[7] J. Mo, Q. Sheng, Li Xue, and S. Zeadally, "RFID Infrastructure Design: A Case Study of Two Australian RFID Projects," IEEE Internet Computing, 13(1), pp. 1421, 2009.

[8] A. Al-Ali, F. Aloul, N. Aji, A. AlZarouni, and N. Fakhro, "Mobile RFID Tracking System," in Proc. of the IEEE International Conference on Information \& Communication Technologies: From Theory to Applications (ICTTA), pp. 1-4, April 2008.

[9] "How much does an RFID tag cost today?" RFID Journal. February 2012. Available at: http://www.rfid journal.com/faq/20.

[10] "Ultracapacitor Offers 75-Foot Read Range for Passive Tags” RFID Journal, June 2011. Available at: http://www.rfidjournal.com/article/view/8565/1.
[11]X. Zhang and M. Tentzeris, "Applications of FastMoving RFID Tags in High-speed Railway Systems,” International Journal of Engineering Business Management, 3(1), pp. 27-31, 2011.

[12] Salik Road Toll. Wikipedia. Available at: http://en.wikipedia.org/wiki/Salik_(road_toll)

[13] "Solar-Powered RFID Reader Measures Road Traffic,” RFID Journal, 2006. Available at: http://www.rfidjourna l.com/article/view/2830.

[14]“RFID Drives Highway Traffic Reports,” 2004. Available at: http://www.rfidjournal.com/article/article print/1243/-1/1/.

[15] CarTEL. Available at: http://cartel.csail.mit.edu/ doku.php.

[16] "Civil Engineers Track Roaming Cell Phones to Monitor Traffic,” 2006. Available at: http://www.aip.org/dbis/IEEE/stories/15139.html

[17]P. Michalopoulos et al. "Vehicle detection through image processing for traffic surveillance and control," United States Patent \#4847772, 1989. 